\title{
Soliton Kinetic Equations with Non-Kolmogorovian Structure: A New Tool for Biological Modeling?
}

\author{
Diederik Aerts*, Marek Czachor ${ }^{\dagger, *}$, Liane Gabora** and Philippe Polk ${ }^{\ddagger}$ \\ ${ }^{*}$ Centrum Leo Apostel (CLEA) and Foundations of the Exact Sciences (FUND) \\ Vrije Universiteit Brussel, 1050 Brussels, Belgium \\ ${ }^{\dagger}$ Katedra Fizyki Teoretycznej i Metod Matematycznych, Politechnika Gdańska, 80-952 Gdańsk, Poland \\ ${ }^{* *}$ Department of Psychology, University of British Columbia, Canada \\ ॠDepartment of Biology, Vrije Universiteit Brussel, 1050 Brussels, Belgium
}

\begin{abstract}
Non-commutative diagrams, where $X \rightarrow Y \rightarrow Z$ is allowed and $X \rightarrow Z \rightarrow Y$ is not, may equally well apply to Malusian experiments with photons traversing polarizers, and to sequences of elementary chemical reactions. This is why non-commutative probabilistic, logical, and dynamical structures necessarily occur in chemical or biological dynamics. We discuss several explicit examples of such systems and propose an exactly solvable nonlinear toy model of a "brain-heart" system. The model involves non-Kolmogorovian probability calculus and soliton kinetic equations integrable by Darboux transformations.
\end{abstract}

Keywords: solitons, Darboux transformations, kinetic equations, non-Kolmogorovian probability, von Neumann equation PACS: 82.39.-k, 03.65.Ca, 05.45.Yv

\section{INTRODUCTION}

This paper can stand on its own as showing how the dynamics of chemical reactions entail a quantum structure even if their microscopic (and obvious) quantum nature is neglected. However, it is most fully appreciated when viewed as part of a large and systematic effort to identify the structure of — quantum, classical or quantum-like — entities and processes in different domains of science. The guiding insight behind our approach is that whether a particular type of entity or process is quantum, classical or quantum-like depends on the nature (quantum, classical or quantum-like) of the structure of the model that describes well the entity or process $^{1}$, and not on the scientific domain to which this entity or process pertains. As such it is for example possible to encounter quantum structure in the macroscopic domain and classical structure in the micro-domain ${ }^{2}$. In this paper we shall show that simple catalytic schemes lead to probabilities having a non-Kolmogorovian structure ${ }^{3}$. In chemistry, when one switches to a dynamical level, one usually arrives at nonlinear kinetic equations. If, however, the governing probabilities are non-Kolmogorovian, as we will show in this paper, one could look for links to nonlinear evolutions of non-Kolmogorovian states or, say, to density matrices if the model is Hilbertian ${ }^{4}$. And indeed, it turns out that nonlinear von Neumann equations may

\footnotetext{
${ }^{1}$ More specifically, we call an entity a "quantum entity" if its behavior is well described by the standard formalism of quantum mecanics, i.e. the formalism as put forward by John von Neumann in [1]. An entity is a classical entity if its behavior is well described by one of the classical mechanics theories. Remark that the theories used in modelling within complexity and chaos approaches are classical mechanical theories. An entity entailing quantum mechanical aspects which cannot be completely modeled within standard quantum mechanics, we call "quantum-like".

${ }^{2}$ In $[2,3]$ a macroscopic entity consisting of a configuration of vessels of water is proposed where Bell inequalities are violated. It has been shown that the violation of Bell inequalities proves the presence of quantum structure, more specifically the presence of a non classical probability structure [4]. Guided by the same idea classical models for quantum spin were elaborated [5, 6], and the structure encountered as such was used to understand better the structural difference between quantum and classical [7]. In section 2 of this paper we consider a chemical reactor and prove that its macroscopic dynamics entails quantum structure. When a classical structure appears in the micro-world one terms it a superselection rule, and is modeled within the standard quantum mechanics formalism in an ad hoc way, more specifically by leaving out the superpositions that would introduce nonclassical structure [8].

${ }^{3}$ One way of showing the presence of quantum structure is by looking at the structure of the probability model that governs the change of the entity. Classical entities and processes entail a probability model that satisfies the axioms of Kolmogorov, and hence is called Kolmogorovian, while the probability model of quantum entities and processes does not satisfy all the axioms of Kolmogorov, and hence is called non-Kolmogorovian.

${ }^{4}$ If a certain structure is non-classical, for example a probability model which is non-Kolmogorovian, a logical first attempt is to try to fit this nonclassical structure into the structure of standard quantum mechanics [1]. Mathematically this comes to looking for a dynamics such as Schrödinger equation on a set of states (of the entity under consideration), where each state is represented by a vector in a complex Hilbert space. A slightly more
} 
be regarded as a "Lax representation" of a classs of integrable nonlinear kinetic equations[16]. This is one of the reasons why one may work with von Neumann-type equations even though the system in question is not "quantum" in the microscopic sense. At the level of Lax representations one can introduce various soliton techniques and find exact solutions. The equations we work with are new in the sense of being simultanously kinetic and solitonic. The result we obtain in this paper fortifies the scheme we worked out in [17], where we introduce a general framework for the description of change-of-state in biological as well as non-biological entities. The framework is referred to as CAP, for context-driven actualization of potential. Processes of change differ with respect to the degree to which they are sensitive to, internalize, and depend upon a particular context, and whether the change of state is deterministic or nondeterministic. The CAP framework has been fruitful for illustrating in broad terms how unusual Darwinian evolution is, and clarifying in what sense change-of-state of living organisms (and also cultural entities) is (and is not) Darwinian. Part of the original goal of the overarching effort was to find and zero in on exactly those areas of biology which have not been successfully analyzed in Darwinian terms and see if formalisms developed for describing change-of-state in other disciplines are instead applicable here. It turns out that, irrespective of the discipline, when change-of-state of an entity depends on how that entity interacts with a context, the resulting probabilities can be non-Kolmogorovian, and the appropriate formalisms for describing this process are either the quantum formalisms or mathematical generalizations of them. This means that our critique within CAP on the Neo-Darwinian Synthesis, where the process of evolution is narrowed down to the interplay of variation and selection, is structural. A process of interplay of variation and selection as imagined within the Neo-Darwinian Synthesis will lead to an underlying probability model which is Kolmogorovian, and in this sense cannot take into account the influence of context as conceived within CAP. In practice it means that the narrowed down evolution process, as within the Neo-Darwinian Synthesis, where only variation and selection play, neglects what happens in the realm of the potential states of the considered entities. It only considers actualized entities, and their actualized interactions, and believes that all of evolution will be steered by these actualized entities, and their actualized interactions. Within CAP also potential states of entities, and potential interactions between these entities will play a role in the process of evolution. In this respect CAP is a generalization of the Neo-Darwinian process, and in this respect furthers the work of scientists who saw the importance of self-organization in biology. Kauffman [18] showed using random Boolean networks, self-organized autocatalytic sets, and related models, that much or perhaps even most change in biological organisms cannot be explained with recourse to Darwin. Others followed suit, and it has meanwhile become evident that neo-Darwinism, powerful though it is, can only to a limited degree account for biological change (see also [19, 20]). The concept of natural selection offers little in the way of explanation for biological forms and phenotypes arise in the first place. (One can ask, for example, if natural selection is such a powerful tool for describing biological change, what can it tell me about the fitness of the offspring I would have with one healthy mate as opposed to another.) Moreover, nonDarwinian processes - such as autopoiesis [21], emergence [22, 23, 24], symbiosis [25], punctuated equilibrium [26] and epigenetic mechanisms [27] — play a vital role. Moreover, the generation of variation is not completely random; convergent pressures are already at work prior to the physical realization of organisms. First, mating is often assortative — mates are chosen on the basis of traits they possess or lack, rather than at random — and relatives are avoided as mates. Second, since Cairns' initial report [28], there is increasing evidence of directed mutation, where the frequency of beneficial mutations is much higher than chance, particularly in environments to which an organism is not well adapted. Furthermore, the concept of fitness, a cornerstone of the neo-Darwinian enterprise, is problematic [29]. In sum, there is more going on in evolution than random variation and natural selection. The CAP framework concentrates on how quantum-like structure enters the realm of biological change. In the present paper we show that this happens already at the level of the dynamics of simple chemical reactions. As one of the applications we describe a situation where we have a composite system whose subsystem is driven by a "pacemaker". Although the driving oscillation is harmonic, the subsystem exhibits characteristic bursting behavior typical of biological oscillators, occcuring due to a nonlinear pacemaker-subsystem coupling. We stress the analogy between the subsystems in question and subsystems ("organs") of a biological dynamical system (to focus attention we refer to the subsystems as the"brain" and the "heart" - a sinusoidal rhythm of the "brain" is translated into bursts of the "heart", and the carrier of information is nonlinear kinetics of a chemical type). Another application is a simple two-qubit system whose evolution possesses three phases separated by "birth" and "death" (before "birth" and after "death" the system consists of two subsystems

general mathematical representation of standard quantum mechanics is the one where the states of the entity are represented by density matrices of a complex Hilbert space, and the dynamics is described by von Neumann equations. This is the representation of standard quantum mechanics that we consider in this paper. Since we need to model non-linear behavior, we however have to consider the non-linear von Neumann equations as studied in $[14,15]$ 
that are effectively noninteracting and evolve independently, and their entropies do not change; the intermediate phase involves exchanges of energy and modifications of entropies of the subsystems).

We begin with a discussion of various non-Kolmogorovian aspects of chemical kinetics. Next, we briefly discuss the links between kinetic equations and their Lax-von Neumann forms. The systems we begin with are those exhibiting rhythmic properties. We introduce their formal description in terms of composite systems and derive an effective equation describing the "heart". This equation can be solved by soliton techniques. We derive the solution and plot the bursts of an oscillating quantity that has a physical meaning of a center of mass of the "heart" (average position of the oscillator). The next section contains a brief discussion of the two-qubit organism, along the lines introduced in[14, 15]. Finally, we end the discussion with explicit examples of kinetic equations associated with von Neumann dymanics. The simplicity and efficiency of the von Neumann formalism as compared with the kinetic equations becomes then especially clear.

\section{NON-KOLMOGOROVIAN ASPECTS OF CHEMICAL KINETICS}

Consider the following catalytic network [16]

$$
\text { (a) } B \stackrel{k}{\rightarrow} A+B, \quad(b) A+X \stackrel{k^{\prime}}{\rightarrow} X+\text { (products), } \quad(c) A+B \stackrel{k}{\rightarrow} A+\text { (products), } \quad(d) Y \stackrel{k^{\prime \prime}}{\rightarrow} B .
$$

Assume that the reaction $(b)$ is of 0 -th order in $A$. This may occur if, for example, $[A]>[X]$ for all $t$, and $X$ catalyzes a decay of $A^{5}$. Let the reaction $(c)$ be of 0 -th order in $B$ (say, if $[B]>[A]$ for all $t$ ) and $A$ catalyzes a decay of $B$. The kinetic equations read

$$
[\dot{A}]=k[B]-k^{\prime}[X], \quad[\dot{B}]=k^{\prime \prime}[Y]-k[A], \quad[\dot{X}]=[\dot{Y}]=0 .
$$

If we define two new (in general non-positive) variables $x=[A]-\left(k^{\prime \prime} / k\right)[Y], y=[B]-\left(k^{\prime} / k\right)[X]$, we can rewrite the system as a harmonic oscillator $i \dot{\xi}=k \xi$ where $\xi=x+i y$. In order to find its "Lax representation", let us introduce the two matrices

$$
H=\left(\begin{array}{cc}
k & 0 \\
0 & 0
\end{array}\right), \quad \rho=\left(\begin{array}{ll}
a & \xi \\
\bar{\xi} & b
\end{array}\right),
$$

$a \geq 0, b \geq 0, a+b>0$. The equations $i \dot{\xi}=k \xi, \dot{a}=\dot{b}=0$ are equivalent to the von Neumann equation $i \dot{\rho}=[H, \rho]$. The equation has the "Lax form" since the right-hand side is given by a commutator. If $\rho>0$ then for any projector $A$ we have $[A]=\operatorname{Tr} \rho A \geq 0$. Let now $A$ and $B$ project, respectively, on $\left(\begin{array}{c}1 \\ 1\end{array}\right)$ and $\left(\begin{array}{c}1 \\ -i\end{array}\right)$. Then $x=[A]-(a+b) / 2$ and $y=[B]-(a+b) / 2$, i.e. $\left(k^{\prime} / k\right)[X]=\left(k^{\prime \prime} / k\right)[Y]=(a+b) / 2$. We thus conclude that the kinetic scheme $(a)-(d)$ implies the dynamics that, for certain initial conditions and appropriately adjusted kinetic constants, is von Neumannian. The kinetic variables satisfy $[A(t)]=\operatorname{Tr} \rho(t) A$ and $[B(t)]=\operatorname{Tr} \rho(t) B$ and the projectors $A$ and $B$ do not commute.

There is also a simpler argument why chemical kinetics is non-Kolmogorovian. There are numerous examples of reactions $X \rightarrow Y$ that cannot happen directly, but involve an intermediate product $Z$. The decomposition into elementary reactions is thus $X \rightarrow Z \rightarrow Y$, and not $X \rightarrow Y$. Formally, the situation is analogous to the Malus law with three or two polarizers.

Finally, consider the chemical reactor shown in Fig. 1 [16, 2]. We fill the bottle with a liquid and perform measurements of its property by means of a beaker. With each of the spigots we associate a random variable, $A$, $A^{\prime}, B$, or $B^{\prime}$, which yields +1 if a given property (e.g. color) is found, and -1 otherwise.

The reactor consists of three chambers, the lowest two are connected by means of a fan whose rotation leads to fluid mixing in the two bottom chambers. Measurements of $A$ or $B$ do not change states of the fluid in the lowest chambers. However, a measurement of either $A^{\prime}$ or $B^{\prime}$ mixes the fluids in the bottom chambers since the fan will rotate due to the flow of the fluid. We assume that the conditions of the experiment allow for collecting some amount of fluid through spigots $A^{\prime}$ or $B^{\prime}$ without noticing the effect of the mixing in the earlier of the two measurements, but the second mesurement will reveal that the fluids got mixed. Fig. 1 symbolically illustrates the structure of the measurement.

\footnotetext{
${ }^{5}$ In chemical notation $[A]$ denotes a nonnegative dynamical variable associated with $A$, usually referred to as a "concentration".
} 
(a)

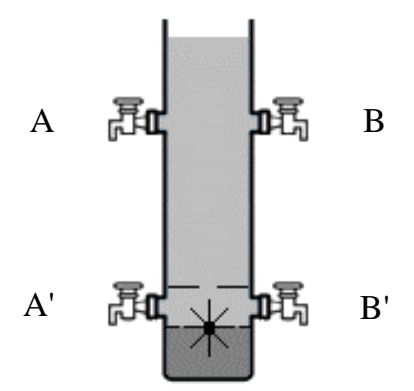

(c)

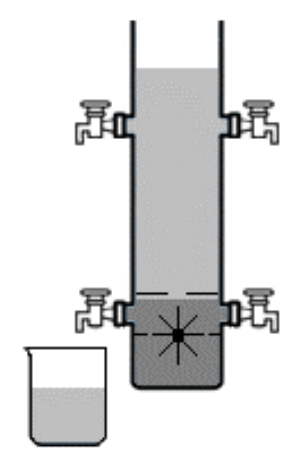

(b)

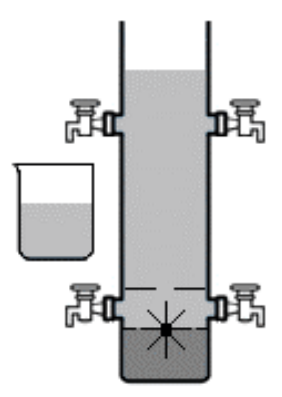

(d)

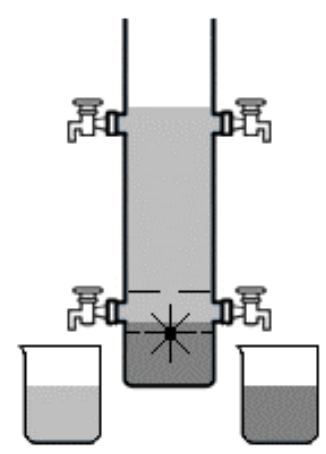


Having one beaker we have to decide on the order of measurements. Clearly, the measurements $A$ and $B$ will always produce +1 (the fluid is red, say), independently of the order in which the four measurements are performed. The same result will be found during the first measurement of either $A^{\prime}$ or $B^{\prime}$. However, once $A^{\prime}$ has been measured, the state of the fluid is changed and a reaction changes the property in question (say, the fluid in the bottom chambers is now green). Therefore, the result of a subsequent measurement of $B^{\prime}$ will produce -1 . An analogous situation is found if one first measures $B^{\prime}$.

The results of possible runs of the experiment can be collected in two quadruples that depend on the order of measurements of $A^{\prime}$ and $B^{\prime}:\left(A, B, A^{\prime}, B^{\prime}\right)=(+1,+1,+1,-1),\left(A, B, B^{\prime}, A^{\prime}\right)=(+1,+1,+1,-1)$. These quadruples can be used to define the random variable $C=A B+A B^{\prime}+A^{\prime} B-A^{\prime} B^{\prime}=1+B^{\prime}+A^{\prime}\left(1-B^{\prime}\right)=2$. The average of $C$ equals $\langle C\rangle=2$. This is consistent with the Bell inequality

$$
\left|\langle A B\rangle+\left\langle A^{\prime} B\right\rangle+\left\langle A B^{\prime}\right\rangle-\left\langle A^{\prime} B^{\prime}\right\rangle\right| \leq 2 .
$$

But what happens if in a single measurement we measure only one of the four product random variables occuring in (4)? Mesurements of $A B, A B^{\prime}$, and $A^{\prime} B$ (performed independently) always give +1 , but the measurement of $A^{\prime} B^{\prime}$ produces -1 . The averages therefore read, $\langle A B\rangle=\left\langle A B^{\prime}\right\rangle=\left\langle A^{\prime} B\right\rangle=+1,\left\langle A^{\prime} B^{\prime}\right\rangle=-1$, and

$$
\langle A B\rangle+\left\langle A^{\prime} B\right\rangle+\left\langle A B^{\prime}\right\rangle-\left\langle A^{\prime} B^{\prime}\right\rangle=4
$$

If all the four random variables are measured on the same reactor than either $A B^{\prime}$ or $A^{\prime} B$ equals -1 because either $A^{\prime}$ or $B^{\prime}$ equals -1 , and we always find $A=B=+1$. If we perform experiments independently than the minus sign can appear only if $A^{\prime}$ and $B^{\prime}$ are measured on the same reactor. All these properties imply that the probability model of the reactor is non-Kolmogorovian. It is interesting that the model is also non-Hilbertian [16].

\section{SOLITON KINETIC EQUATIONS}

The linear von Neumann equation occured in the previous section as a "Lax form" of a system of kinetic equations associated with certain catalytic reactions. Linearity was implied by the fact that reactions were of zero order. However, the generic case one finds in biochemical systems does not involve zero-order reactions and, hence, is nonlinear. As one of the simple generalizations of the von Neumann equation one may consider its nonlinear version

$$
i \dot{\rho}=[H, f(\rho)]
$$

introduced in [30] in the context of nonlinear quantum mechanics. There are reasons to assume that $f(\rho)$ is an operator function satisfying the "no-feedback, no-nonlinearity" property, formally meaning that $f(\rho)=\rho$ if $\rho^{2}=\rho[15,31]$. The latter condition is fulfilled, up to rescaling of time, by any polynomial; also any function satisfying $f(0)=0$, $f(1)=1$ will do the job since by spectral theorem it will map a projecor into itself.

It is important that real and imaginary parts of all matrix elements of $\rho$ can be directly associated with probabilities. The construction is simple. Consider an operator $H$ that possesses a discrete part of spectrum, and let $H|j\rangle=E_{j}|j\rangle$, $\langle j \mid k\rangle=\delta_{j k}$, and $\rho_{j k}=\langle j|\rho| k\rangle=x_{j k}+i y_{j k}$. Now, let $|j k\rangle=(|j\rangle+|k\rangle) / \sqrt{2},\left|j k^{\prime}\right\rangle=(|j\rangle-i|k\rangle) / \sqrt{2}, P_{j k}=|j k\rangle\langle j k|, P_{j k}^{\prime}=$ $\left|j k^{\prime}\right\rangle\left\langle j k^{\prime}\left|, P_{j}=\right| j\right\rangle\langle j|$. Then $x_{j k}=p_{j k}-\frac{1}{2} p_{j}-\frac{1}{2} p_{k}, y_{j k}=p_{j k}^{\prime}-\frac{1}{2} p_{j}-\frac{1}{2} p_{k}$, where $p_{k}=\operatorname{Tr} P_{k} \rho=\rho_{k k}, p_{j k}=\operatorname{Tr} P_{j k} \rho$, $p_{j k}^{\prime}=\operatorname{Tr} P_{j k}^{\prime} \rho$. The latter formulas imply that $0 \leq p_{k}, p_{j k}(t), p_{j k}^{\prime}(t) \leq 1$, for any $t$, and thus are some probabilities. The probabilities $p_{k}$ are time independent. If $f(\rho)$ is a polynomial then (6) is equivalent to a set of nonlinear kinetic equations with polynomial nonlinearities. The system is conservative if $t$ is real. One can add dissipation by replacing $t$ with a complex parameter. In particular, an imaginary time turns our equation into a kind of heat equation.

Still, we have more than just a set of nonlinear kinetic equations. The probabilities are rooted in a nonclassical (Hilbert-space) model of probability so that we have much more control over the structure of the probabilities in question. In particular, we can derive certain uncertainty relations between different probabilities. This is not surprising if one thinks of the chemical reactor violating the Bell inequality where manipulations with the spigots led to noncommuting random variables. For any three operators satisfying $[A, B]=i C$ one can prove $\Delta A \Delta B \geq \frac{1}{2}|\langle C\rangle|$ where $\Delta A=\sqrt{\left\langle A^{2}\right\rangle-\langle A\rangle^{2}},\langle A\rangle=\operatorname{Tr}(A \rho)$, etc. If $A=P=P^{2}$ is a one-dimensional projector then $\langle P\rangle=p$ is a probability and one finds $\Delta P=\sqrt{p(1-p)}$. We say that two propositions $P_{1}$ and $P_{2}$ are complementary if $\Delta P_{1} \Delta P_{2} \geq \varepsilon>0$.

In order to show that the propositions, say $P_{j}$ and $P_{j k}$, are complementary, we compute

$$
\left[P_{j}, P_{j k}\right]=\frac{1}{2}(|j\rangle\langle k|-| k\rangle\langle j|)=i C
$$

Soliton Kinetic Equations with Non-Kolmogorovian Structure: A New Tool for Biological Modeling?October 13, 20055 
and $\langle C\rangle=y_{k j}$. Finally,

$$
\sqrt{p_{j}\left(1-p_{j}\right)} \sqrt{p_{j k}\left(1-p_{j k}\right)} \geq \frac{1}{2}\left|y_{k j}\right| .
$$

The variable $y_{k j}$ measures complementarity of $P_{j}$ and $P_{j k}$. The complementarity varies in time, analogously to the morphogenesis of complementarity discussed in [15]. The fact that complementarity should be time dependent was also clear from the reactor example.

Another important property of the dynamics is that (6) is integrable in the sense of soliton theory for any $f$. A soliton technique of solving (6), based on Darboux transformations, was introduced in [14], and further developed in $[32,33,34]$. Therefore, as opposed to standard kinetic equations that typically have to be solved numerically, we can work with exact analytic solutions.

\section{BIOLOGICAL OSCILLATORS, COUPLED SYSTEMS, AND RHYTHMIC PHENOMENA}

If one looks for the most striking manifestations of biochemical nonlinear evolutions one arrives at phenomena that are rhythmic. Periodic switching, with periods ranging from fraction of a second to years, is encountered at all levels of biological organization, and its successful modelling in terms of nonlinear kinetic equations is one of the great achievements of computational biology (for reviews cf. [35, 36]). Of particular interest are theoretical studies of circa-rhythms. Let us recall that circa-rhythms are "classes of rhythms that are capable of free-running in constant conditions with a period approximating that of the environmental cycle to which they are normally synchronized" [37]. The examples of the rhythms are the circadian (24 hours), the circalunar (28 days), or the circannual (365.25 days) ones. What makes circa-rhythms interesting from a formal point of view is the interplay between the phases where there exists an external forcing (light-darkness periods, say), and the rhythmicity that sustains even after the external driving is switched off (as in experiments in constant darkness). Formally, the two cases may correspond to nonlinearities involving (or not) an explicit time dependence of some parameters.

The models are typically finite-dimensional (as dynamical systems) and involve nonlinearities of a step-function type. The latter are convenient from the point of view of "engineering" of a nonlinear behavior. Having some experience with nonlinear equations one can force a system to behave in a given way, at least within a given range of parameters. To give an example, the model of circadian rhythms in Neurospora [38] involves four variables and two types of Hill functions. The rhythmicity occurs in the model even without an external driving, but the case with explicit time dependence of coefficients is treated as well. Similar constructions are given in [39, 40].

As one of the first applications of (6) in a biological context we thus address the problem of circa-rhythmicity. The main idea is to consider a composite Hamiltonian system that, as a whole, is conservative but not isolated from the external world (let us term the system an organism; our organism will not "live" if we isolate it from the external world!). We take simple and generic polynomial nonlinearities, and do not try to force switching by means of Hill-type functions. The lack of isolation of the organism from the environment means that it has nontrivial correlations with the outside world, a fact that allows for starting with an initial condition $\rho(0) \neq \rho(0)^{2}$. Only in such a case the nonlinearity inherent to the organism is nontrivial. There are two sets of degrees of freedom corresponding to two subsystems of the organism (let us term them the "brain" and the "heart"). When we look at certain averages associated with one of the subsystems (the heart) we find that their time evolution can be determined by an effective density matrix whose dynamics is given again by an equation of the form (6), but now with explicitly time-dependent coefficients in the nonlinear function $f(\rho)$. Therefore, the interaction between the brain and the heart effectively turns the brain into a pacemaker that drives the heart and generates its evolution with characteristic bursting patterns.

Although the "brain" and the "heart" may be treated as metaphores and the model given below is to a large extent a toy one, we are inclined to defend a more straightforward interpretation of our construction. One has to keep in mind that the reactor we have described in the previous section is a highly non-Kolmogorovian system due to various interactions between its different parts. There is no reason to believe that a real biochemical system is less non-Kolmogorovian. The assumptions we make about the "brain" and the "heart" mean that the system is nonKolmogorovian and Hilbertian. The latter is perhaps even too weak a postulate, but yet this is a toy model. 


\section{FORMAL DESCRIPTION: DYNAMICS IN A SUBSYSTEM}

So let us consider a composite system consisting of two subsystems interacting with each other via a nonlinear coupling defined by a function $f(\rho)$ satisfying the "no-feedback, no nonlinearity" condition. As a simple example consider

$$
i \dot{\vartheta}=\left[\omega(\underbrace{b_{1}^{\dagger} b_{1}-b_{2}^{\dagger} b_{2}}_{J})+(1+X) a^{\dagger} a, \vartheta\right]-\left[X a^{\dagger} a, f(\vartheta)\right]
$$

where $\left[b_{k}, b_{k}^{\dagger}\right]=1=\left[a, a^{\dagger}\right]$ and $X=b_{1}+b_{1}^{\dagger}+b_{2}+b_{2}^{\dagger}$. For $\vartheta^{2}=\vartheta$ we find $i \dot{\vartheta}=\left[\omega J+a^{\dagger} a, \vartheta\right]$ describing three independent degrees of freedom: Harmonic oscillation with unit frequency (the heart) combined with rotation in a plane with frequency $\omega$ (the brain). For $\vartheta^{2} \neq \vartheta$ rotation and oscillation get nonlinearly coupled. Let us now eliminate the rotation by switching to a rotating reference frame $\rho=e^{i \omega J t} \vartheta e^{-i \omega J t}$ where the density matrix satisfies

$$
i \dot{\rho}=\left[(1+X(t)) a^{\dagger} a, \rho\right]-\left[X(t) a^{\dagger} a, f(\rho)\right]
$$

Denoting $Y=-i\left(b_{1}+b_{2}^{\dagger}-b_{1}^{\dagger}-b_{2}\right)$ we find

$$
X(t)=e^{i \omega J t} X e^{-i \omega J t}=X \cos \omega t+Y \sin \omega t, \quad Y(t)=e^{i \omega J t} Y e^{-i \omega J t}=Y \cos \omega t-X \sin \omega t
$$

and, for any $t$ and $t^{\prime},\left[X(t), X\left(t^{\prime}\right)\right]=\left[Y(t), Y\left(t^{\prime}\right)\right]=\left[X(t), Y\left(t^{\prime}\right)\right]=0$. Since $X, Y$, and $a^{\dagger} a$ commute we can introduce their joint eigenvectors

$$
\begin{aligned}
& X|x, y, n\rangle=x|x, y, n\rangle, \quad Y|x, y, n\rangle=y|x, y, n\rangle, \quad X(t)|x, y, n\rangle=x(t)|x, y, n\rangle, \quad Y(t)|x, y, n\rangle=y(t)|x, y, n\rangle, \\
& a^{\dagger} a|x, y, n\rangle=n|x, y, n\rangle, \quad x(t)=x \cos \omega t+y \sin \omega t, \quad y(t)=y \cos \omega t-x \sin \omega t .
\end{aligned}
$$

Now take any time-independent normalized vector $|\psi\rangle=\int d x d y \psi(x, y)|x, y\rangle$ and make the Ansatz $\rho(t)=|\psi\rangle\langle\psi| \otimes$ $w(t)$ where $w$ is a density matrix acting only on the oscillator degress of freedom. Denoting $g(\rho)=\rho-f(\rho)=$ $|\psi\rangle\langle\psi| \otimes g(w)$ we rewrite (10) as

$$
|\psi\rangle\langle\psi|\otimes i \dot{w}=| \psi\rangle\left\langle\psi\left|\otimes\left[a^{\dagger} a, w\right]+X(t)\right| \psi\right\rangle\left\langle\psi\left|\otimes a^{\dagger} a g(w)-\right| \psi\right\rangle\langle\psi| X(t) \otimes g(w) a^{\dagger} a
$$

Taking matrix elements of both sides of (13) between arbitrary $\langle x, y|$ and $\left|x^{\prime}, y^{\prime}\right\rangle$ we obtain

$$
\psi(x, y) \overline{\psi\left(x^{\prime}, y^{\prime}\right)} i \dot{w}=\psi(x, y) \overline{\psi\left(x^{\prime}, y^{\prime}\right)}\left[a^{\dagger} a, w\right]+\psi(x, y) \overline{\psi\left(x^{\prime}, y^{\prime}\right)}\left(x(t) a^{\dagger} a g(w)-x^{\prime}(t) g(w) a^{\dagger} a\right) .
$$

The Ansatz is internally consistent only if $\psi(x, y)=\sqrt{\delta\left(x-x_{0}\right) \delta\left(y-y_{0}\right)}$, and then the diagonal $x=x^{\prime}=x_{0}, y=y^{\prime}=y_{0}$ leads to

$$
\begin{aligned}
i \dot{w} & =\left[a^{\dagger} a, w\right]-x_{0}(t)\left[a^{\dagger} a, g(w)\right]=\left[a^{\dagger} a, \tilde{f}(w)\right], \quad x_{0}(t)=x_{0} \cos \omega t+y_{0} \sin \omega t \\
\tilde{f}(w) & =w-x_{0}(t) g(w)=\left(1-x_{0}(t)\right) w+x_{0}(t) f(w) .
\end{aligned}
$$

If $f$ satisfies the "no feedback, no nonlinearity" condition $f(\rho)=\rho$ for $\rho^{2}=\rho$, the same holds for the effective $\tilde{f}$. Eq. (15) determines the effective dynamics of the subsystem, and belongs to the class of Darboux-integrable nonlinear von Neumann equations. It can be explicitly integrated by means of the techniques introduced in [14, 32].

\section{QUANTUM PACEMAKER AND FEEDBACK}

The pacemaker is an oscillatory system whose state can be modified by an external entraining agent, a zeitgeber [37]. In our example the pacemaker corresponds to the part of the composite system described by the Hamiltonian $\omega J$, i.e. to the brain, and the zeitgeber can be associated with the world external to the organism. Our main interest in this example is in the free-running oscillation of the pacemaker and the free-running rhythms of the heart it induces.

The next level is the coupling between the pacemaker, which defines the clock "mechanism" of the brain, and the observable-level circa-rhythms which define the "hands" of the clock (center of mass of the heart). We assume a nonlinear feedback between the hands and the mechanism, but the dynamics is nondissipative in the sense that the 
energy of the hands averaged over a single cycle of the oscillation is constant. Motivated by the analysis of the previous section we concentrate on the following class of equations

$$
i \dot{w}(t)=f_{1}(t)[H, w(t)]+f_{2}(t)[H, f(w(t))]
$$

which define the state of the hands of the clock. The "no-feedback, no-nonlinearity" condition reads $w(t)=f_{1}(t) w(t)+$ $f_{2}(t) f(w(t))$ whenever $w(t)^{2}=w(t)$. Now assume we know a solution $w_{0}(t)$ of (6). Then

$$
w(t)=e^{-i H \int_{0}^{t} f_{1}(x) d x} w_{0}\left(\int_{0}^{t} f_{2}(x) d x\right) e^{i H \int_{0}^{t} f_{1}(x) d x}
$$

is a solution of (17) as can be verified by a direct calculation. The whole problem of solving (17) reduces to finding a solution of (6), which can be performed by soliton techniques [14, 32].

Let us consider a simple but generic example where $f(w)=(1-s) w+s w^{2}$ and

$$
f_{1}(t)=1+\varepsilon \cos \omega t, \quad f_{2}(t)=-\varepsilon \cos \omega t
$$

The parameter $s$ allows us to compare situations where the driven dynamics is linear $(s=0)$ and purely nonlinear $(s=1)$, and for the two cases investigate the role of the $\varepsilon$ s. Varying $s$ we can also investigate stability properties of the rhythms under fluctuations of the feedback.

\section{THE HEART: HANDS OF THE CLOCK}

The hands of the clock are described by the Hamiltonian $H=a^{\dagger} a=\sum_{n=0}^{\infty} n|n\rangle\langle n|$ of a harmonic oscillator type. The frequency of the oscillator is equal to unity, meaning that this is a reference frequency for all the other frequencies found in the system. Modelling the heart by an oscillator is quite natural for obvious reasons. The quantum oscillator has in addition the appealing property of being delocalized, or extended in some formal sense. Intuitively, in our model, the hands move at time $t$ to the region of space of greatest concentration of probability density. The probability density may be regarded as a measure of state of the heart. In the absence of a feedback between the hands and the pacemaker, the hands oscillate harmonically with their own internal frequency (unless damping is added). We shall see later that the nonlinear coupling may practically suppress the internal oscillations of the hands at certain intervals of time. What will remain are the sudden bursts (or "heart-beats") occuring, roughly, with the period of the pacemaker.

Quantum harmonic oscillator is an infinite dimensional dynamical system and, hence, a solution of von Neumann equations may be characterized by an arbitrary number of parameters determining the initial state of the heart. There exists a simple trick allowing to construct infinitely-dimensional solutions on the basis of a single finite-dimensional one. The trick exploits equal spacing of eigenvalues of $H$. With our choice of units the eigenvalues are given simply by natural numbers. Let us divide them into sets containing $N$ elements: $\{0,1, \ldots, N-1\},\{N, N+1, \ldots, N+N-1\}$, and so on. Each such a subset corresponds to a block in the Hamiltonian, and each block can be represented by a $N \times N$ diagonal matrix of the form

$$
H_{k}=k 1+\operatorname{diag}(0,1, \ldots, N-1)=k 1+H_{0} .
$$

As a consequence, in each block we have to solve the same matrix equation since a restriction $w_{k}$ of $w$ to the $k$-th subspace satisfies

$$
i \dot{w}_{k}=\left[H_{k}, f\left(w_{k}\right)\right]=\left[H_{0}, f\left(w_{k}\right)\right] .
$$

The job can be reduced to finding a sufficiently general solution of a $N \times N$ problem. In each subspace we can take a different initial condition and a different normalization of trace. The whole infinite-dimensional solution will take the form of a direct sum

$$
w(t)=\oplus_{k=0}^{\infty} p_{k} w_{k}\left(t, p_{k}\right)
$$

$\sum_{k=0}^{\infty} p_{k}=1$. The $k$-th part depends on $p_{k}$ in a complicated way since the function $f(w)$ is not 1 -homogeneous, i.e. $f(\lambda w) \neq \lambda f(w)$. The inhomogeneity implies that change of normalization simultaneously rescales time; the normalization of probability implies that a change of $p_{k}$ in a $k$-th subspace influences all the other subspaces by 
making their dynamics faster or slower. In this sense the solution, in spite of its simplicity, is not a simple direct sum of independent evolutions.

In order to illustrate the possible effects we can use the solutions derived in [15] for the simplest nontrivial case involving self-switching, i.e. for $N=3$ and quadratic nonlinearity (nonlinearity that did not explicitly depend on time). We select a subspace spanned by three subsequent vectors $|k\rangle,|k+1\rangle,|k+2\rangle$. The family of interest is parametrized by $\alpha \in \mathbf{R}$ controlling the "moment" and type of switching between bursts. The parameter naturally occurs at the level of the Darboux transformation, where it characterizes an initial condition for the solution of the Lax pair. The density matrices $w_{k}(t)=\sum_{m, n=0}^{2} w_{m n}|k+m\rangle\langle k+n|$ are completely characterized by the $k$-independent matrix of timedependent coefficients $w_{m n}$. The reader may check by a straightforward substitution ${ }^{6}$ that the matrix

$$
\left(\begin{array}{lll}
w_{00} & w_{01} & w_{02} \\
w_{10} & w_{11} & w_{12} \\
w_{20} & w_{21} & w_{22}
\end{array}\right)=\frac{1}{15+\sqrt{5}}\left(\begin{array}{ccc}
5 & \xi(t) & \zeta(t) \\
\bar{\xi}(t) & 5+\sqrt{5} & \xi(t) \\
\bar{\zeta}(t) & \bar{\xi}(t) & 5
\end{array}\right)
$$

with

$$
\xi(t)=\frac{(2+3 i-\sqrt{5} i) \sqrt{3+\sqrt{5}} \alpha}{\sqrt{3}\left(e^{\gamma t}+\alpha^{2} e^{-\gamma t}\right)} e^{i \omega_{0} t}, \quad \zeta(t)=-\frac{9 e^{2 \gamma t}+(1+4 \sqrt{5} i) \alpha^{2}}{3\left(e^{2 \gamma t}+\alpha^{2}\right)} e^{2 i \omega_{0} t}
$$

is indeed a normalized $(\operatorname{Tr} w=1)$ solution of the von Neumann equation $i \dot{w}=\left[H,(1-s) w+s w^{2}\right], H=\operatorname{diag}(0,1,2)$. The parameters are $\omega_{0}=1-\frac{5+\sqrt{5}}{15+\sqrt{5}} s, \gamma=\frac{2}{15+\sqrt{5}} s$. Now let us rescale the trace. We do it in three steps. The modified density matrix $w_{1}(t)=e^{i(1-s) H t} w(t) e^{-i(1-s) H t}$ is a solution of $i \dot{w}_{1}(t)=\left[s H, w_{1}(t)^{2}\right]$. Therefore

$$
w_{2}(t)=\Lambda e^{i(1-s) \Lambda H t} w(\Lambda t) e^{-i(1-s) \Lambda H t}
$$

is also a solution of $i \dot{w}_{2}(t)=\left[H, s w_{2}(t)^{2}\right]$ and $w_{3}(t)=e^{-i(1-s) H t} w_{2}(t) e^{i(1-s) H t}$ solves

$$
i \dot{w}_{3}(t)=\left[H,(1-s) w_{3}(t)+s w_{3}(t)^{2}\right] .
$$

Performing these operations on our explicit solution we find

$$
w_{3}(t)=\frac{\Lambda}{15+\sqrt{5}}\left(\begin{array}{ccc}
5 & \xi_{3}(t) & \zeta_{3}(t) \\
\bar{\xi}_{3}(t) & 5+\sqrt{5} & \xi_{3}(t) \\
\bar{\zeta}_{3}(t) & \bar{\xi}_{3}(t) & 5
\end{array}\right)
$$

with

$\xi_{3}(t)=\frac{(2+3 i-\sqrt{5} i) \sqrt{3+\sqrt{5}} \alpha}{\sqrt{3}\left(e^{\gamma \Lambda t}+\alpha^{2} e^{-\gamma \Lambda t}\right)} e^{i\left(\omega_{0} \Lambda+(1-s)(1-\Lambda)\right) t}, \quad \zeta_{3}(t)=-\frac{9 e^{2 \gamma \Lambda t}+(1+4 \sqrt{5} i) \alpha^{2}}{3\left(e^{2 \gamma \Lambda t}+\alpha^{2}\right)} e^{2 i\left(\omega_{0} \Lambda+(1-s)(1-\Lambda)\right) t}$,

Now $\int_{0}^{t} f_{1}(x) d x=t+\frac{\varepsilon}{\omega} \sin \omega t, \int_{0}^{t} f_{2}(x) d x=-\frac{\varepsilon}{\omega} \sin \omega t$. We finally obtain the solution $w(t)$ of

$$
i \dot{w}(t)=(1+\varepsilon \cos \omega t)[H, w(t)]-\varepsilon \cos \omega t\left[H,(1-s) w(t)+s w(t)^{2}\right] .
$$

Explicitly,

$$
\begin{aligned}
w(t) & =\frac{\Lambda}{15+\sqrt{5}}\left(\begin{array}{ccc}
5 & \xi(t) & \zeta(t) \\
\bar{\xi}(t) & 5+\sqrt{5} & \xi(t) \\
\bar{\zeta}(t) & \bar{\xi}(t) & 5
\end{array}\right) \\
\xi(t) & =\frac{(2+3 i-\sqrt{5} i) \sqrt{3+\sqrt{5}} \alpha}{\sqrt{3}\left(e^{-\gamma \Lambda \frac{\varepsilon}{\omega} \sin \omega t}+\alpha^{2} e^{\gamma \Lambda \frac{\varepsilon}{\omega} \sin \omega t}\right)} e^{-i\left(\varepsilon \omega_{0} \Lambda+\varepsilon(1-s)(1-\Lambda)-\varepsilon\right) \frac{\sin \omega t}{\omega}} e^{i t} \\
\zeta(t) & =-\frac{9 e^{-2 \gamma \Lambda \frac{\varepsilon}{\omega} \sin \omega t}+(1+4 \sqrt{5} i) \alpha^{2}}{3\left(e^{-2 \gamma \Lambda \frac{\varepsilon}{\omega} \sin \omega t}+\alpha^{2}\right)} e^{-2 i\left(\varepsilon \omega_{0} \Lambda+\varepsilon(1-s)(1-\Lambda)-\varepsilon\right) \frac{\sin \omega t}{\omega}} e^{2 i t}
\end{aligned}
$$

\footnotetext{
6 The solutions were checked by means of Mathematica 4.2 .
}

Soliton Kinetic Equations with Non-Kolmogorovian Structure: A New Tool for Biological Modeling?October 13, 20059 


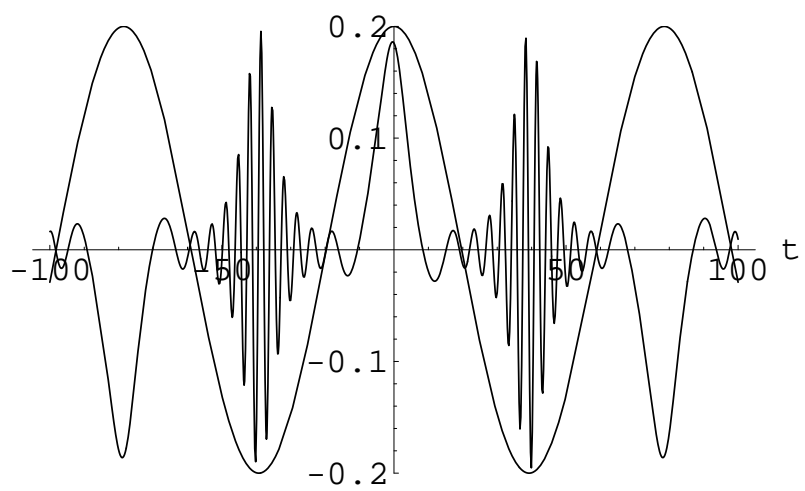

FIGURE 2. Bursts of average position $\operatorname{Tr} \hat{q} w(t)$ of the heart for $\varepsilon=2, \omega=0.08, \alpha=1, s=-1.1, \Lambda=1$. The brain pacemaker sinusoidal oscillation is shown as a reference.

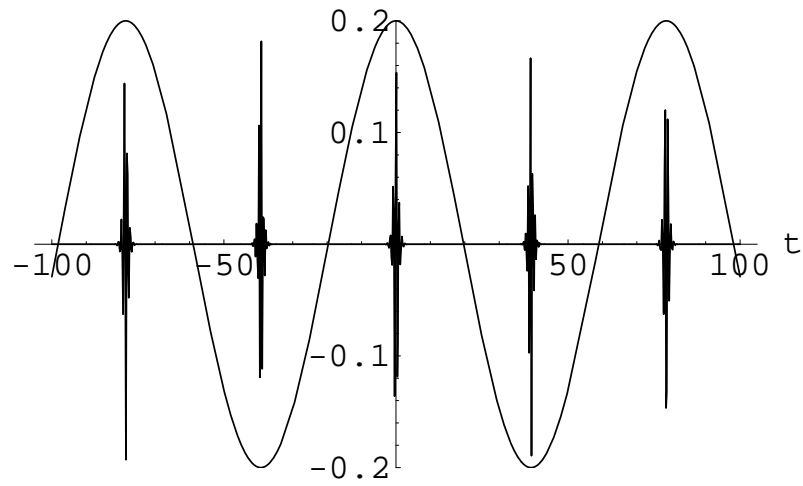

FIGURE 3. The same parameters as in Fig. 2 but with $s=-10$, i.e. for stronger brain-heart coupling.

Since $\operatorname{Tr} H w(t)=\operatorname{Tr} H w(0), \operatorname{Tr} H w(t)^{2}=\operatorname{Tr} H w(0)^{2}$, the internal energy of the system, averaged over one period $T$ of the pacemaker oscillation, is

$$
E=\frac{1}{T} \int_{t}^{t+T} d t^{\prime} \operatorname{Tr} H f\left(w\left(t^{\prime}\right)\right)=\operatorname{Tr} H w(0)
$$

and does not depend on $t$. In this sense the subsystem is conservative.

If one does not integrate over the pacemaker period, one finds that the internal energy of the hands harmonically oscillates with the pacemaker frequency $\omega$. What is characteristic, however, the hands do not oscillate harmonically but behave as if they were accumulating energy during the phases of quiescence in order to suddenly release it in violent bursts.

In Figs. 2-5 we plot the dynamics of the hands $\bar{q}(t)=\operatorname{Tr} \hat{q} w(t)=\int_{-\infty}^{\infty} d q q\langle q|w(t)| q\rangle$ as functions of time for different parameters characterizing the nonlinearity, and for different initial conditions. In Fig. 6 we analyze stability of the bursts of the heart under changes of the brain-heart coupling. The system is very stable: Even very large changes of the parameter $s$ do not change the qualitative structure of the bursts. Only for $s$ sufficiently close to 0 the bursts decay into periodic oscillations whose frequency is unrelated to the one of the brain pacemaker.

\section{SCENES FROM THE LIFE OF A TWO-QUBIT ORGANISM}

In this example there is no external driving by a pacemaker. The system consists of two subsystems that as a whole interact via nonlinearity. The system is conservative, i.e. both its average energy and entropy are constant. We will see that one can split the history of the system into three effective phases. First, the phase where the two subsystems behave as if they were completely uncoupled. Then, the two parts start to exhibit certain joint activity, there are flows 


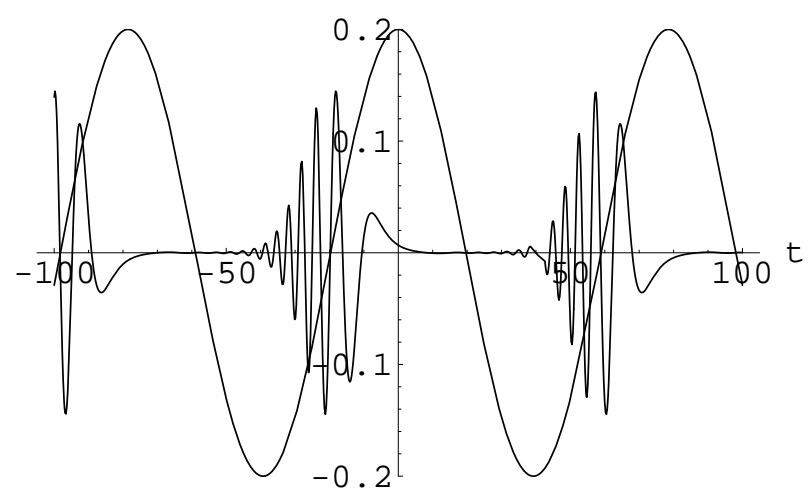

FIGURE 4. The same parameters as in Fig. 2 but with $\alpha=e^{-4}$.

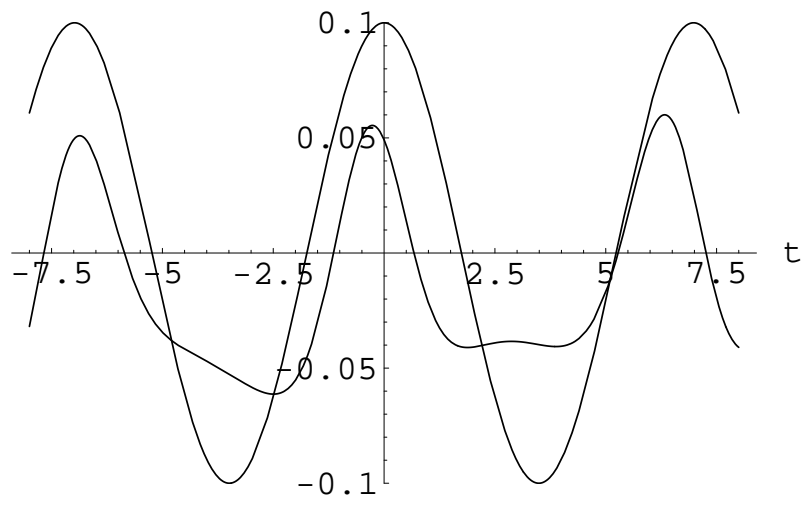

FIGURE 5. $\operatorname{Tr} \hat{q} w(t)$ for $\varepsilon=2, \omega=0.9, \alpha=e^{2}, s=1, \Lambda=1$. The sinusoidal oscillation is the pacemaker.

of energy between the subsystems, and the entropies of the subsystems change. Finally, the activity dies out and the system becomes indistinguishable from the one that never involved any internal activity. Analogies to "birth", "life", and "death" are so striking that the name "organism" becomes even more justified.

The details of the model are taken from [15]. The two-qubit system is described by the Hamiltonian

$$
H=2 \sigma_{x} \otimes 1+1 \otimes \sigma_{z} .
$$

We start with the unnormalized density matrix

$$
\rho(0)=\frac{1}{2}\left(\begin{array}{cccc}
5+\sqrt{7} & 0 & 0 & 0 \\
0 & 5-\sqrt{7} & 0 & 0 \\
0 & 0 & 5+\sqrt{15} & 0 \\
0 & 0 & 0 & 5-\sqrt{15}
\end{array}\right)
$$

which is written in such a basis that

$$
H=\left(\begin{array}{cccc}
1 & 2 & 0 & 0 \\
2 & 1 & 0 & 0 \\
0 & 0 & -1 & 2 \\
0 & 0 & 2 & -1
\end{array}\right)
$$

The density matrix

$$
\rho(t)=\exp [-5 i H t] \rho(0) \exp [5 i H t]
$$




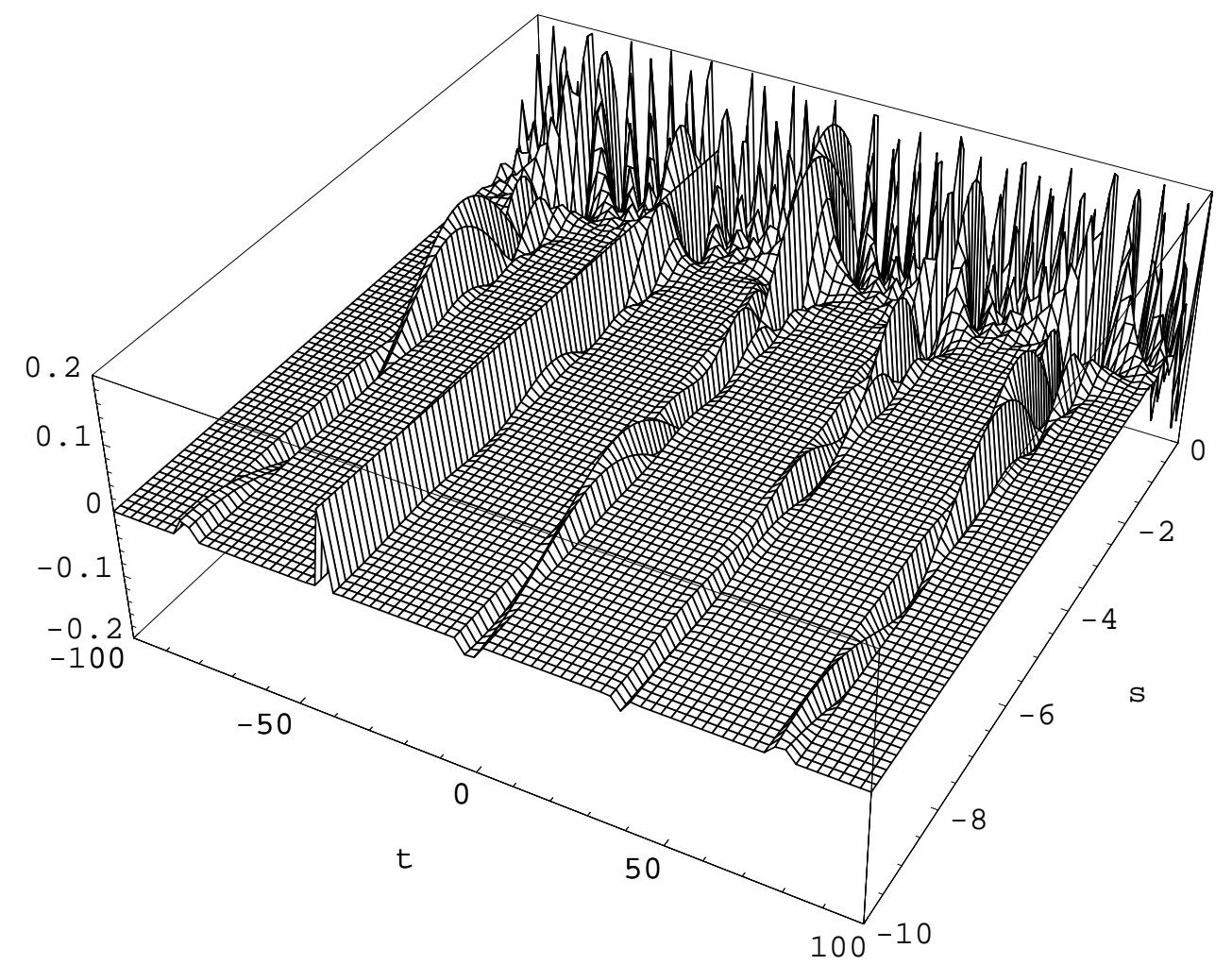

FIGURE 6. Stability of the heart-beats under changes of nonlinear coupling with the brain. Average position $\operatorname{Tr} \hat{q} w(t)$ for $\varepsilon=2$, $\omega=0.08, \alpha=1, \Lambda=1$, and $-10 \leq s \leq 0$. For $s$ sufficiently far from 0 the bursts do not qualitatively change with fluctuations of $s$. Fine details of the bursts are smeared out by coarse-graining of the plot.

is a solution of (6) with $f(\rho)=\rho^{2}$. Such a $\rho(t)$ describes simultaneously a dynamics of two non-interacting systems satisfying the linear von Neumann equation

$$
i \dot{\rho}=5\left[2 \sigma_{x} \otimes 1+1 \otimes \sigma_{z}, \rho\right] .
$$

The environment does not trigger in this solution any switching, but only makes its evolution five times faster than in the absence of the feedback. The Darboux transformation when applied to $\rho(t)$ produces the solution

$$
\rho_{1}(t)=\exp [-5 i H t] \rho_{\text {int }}(t) \exp [5 i H t]
$$

where

$$
\rho_{\text {int }}(t)=\frac{1}{2}\left(\begin{array}{cccc}
5-\sqrt{7} \tanh 2 t & 0 & \frac{-13 i-3 \sqrt{7}-\sqrt{15}-i \sqrt{105}}{8 \operatorname{cosh2t}} & \frac{-7 i+3 \sqrt{7}-3 \sqrt{15}+i \sqrt{105}}{8 \cosh 2 t} \\
0 & 5+\sqrt{7} \tanh 2 t & \frac{15 i+\sqrt{7}-\sqrt{15}-i \sqrt{105}}{8 \cosh 2 t} & \frac{\sqrt{7}+\sqrt{15}}{2 \cosh 2 t} \\
\frac{13 i-3 \sqrt{7}-\sqrt{15}+i \sqrt{105}}{8 \cosh 2 t} & \frac{-15 i+\sqrt{7}-\sqrt{15}+i \sqrt{105}}{8 \cosh 2 t} & 5+\sqrt{15} \tanh 2 t & 0 \\
\frac{7 i+3 \sqrt{7}-3 \sqrt{15}-i \sqrt{105}}{8 \cosh 2 t} & \frac{\sqrt{7}+\sqrt{15}}{2 \cosh 2 t} & 0 & 5-\sqrt{15} \tanh 2 t
\end{array}\right) .
$$

The switching between the two asymptotic evolutions is triggered in the neighborhood of $t=0$.

If we look at the subentities forming the organism we notice that they do not evolve independently. The easiest way of seeing this is to compute eigenvalues of reduced density matrices. Both subsystems are two-dimensional so there are two eigenvalues for each reduced density matrix. They read

$$
\begin{aligned}
& p_{ \pm}(1)=\frac{1}{2} \pm \frac{\sqrt{15}-\sqrt{7}}{20} \tanh 2 t, \quad \text { qubit } 1 \\
& p_{ \pm}(2)=\frac{1}{2} \pm \frac{\sqrt{26+2 \sqrt{105}}}{40 \cosh 2 t}, \quad \text { qubit } 2 .
\end{aligned}
$$

Soliton Kinetic Equations with Non-Kolmogorovian Structure: A New Tool for Biological Modeling?October 13, 200512 


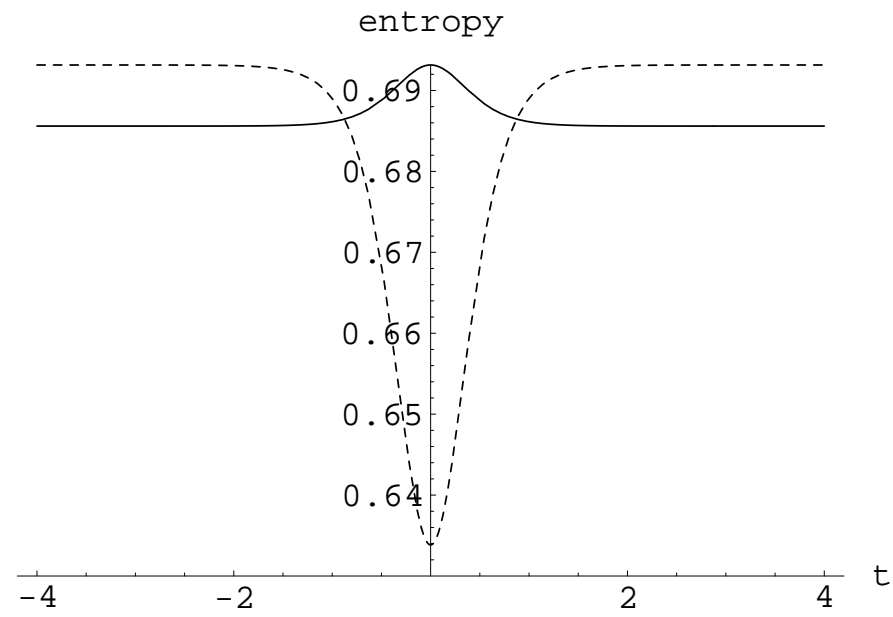

FIGURE 7. Entropies of the two parts of the two-qubit organism as functions of time. The organism lives, approximately, for $-2<t<2$.

The asymptotics are

$$
\begin{aligned}
\rho_{\text {int }}(-\infty)= & \frac{1}{2}\left(\begin{array}{cccc}
5-\sqrt{7} & 0 & 0 & 0 \\
0 & 5+\sqrt{7} & 0 & 0 \\
0 & 0 & 5-\sqrt{15} & 0 \\
0 & 0 & 0 & 5+\sqrt{15}
\end{array}\right), \\
\rho_{\text {int }}(+\infty)= & \frac{1}{2}\left(\begin{array}{cccc}
5+\sqrt{7} & 0 & 0 & 0 \\
0 & 5-\sqrt{7} & 0 & 0 \\
0 & 0 & 5+\sqrt{15} & 0 \\
0 & 0 & 0 & 5-\sqrt{15}
\end{array}\right)=\rho(0),
\end{aligned}
$$

and therefore the dynamics represents asymptotically two non-interacting subentities. It is also interesting that the $+\infty$ asymptotics is $\rho_{1}(t) \approx \rho(t)$. At large times the "organism" that "dies" becomes practically indistinguishable from the one that never "lived".

The "life" of the organism is the period of time when the two subentities exhibit certain joint activity. Computing the von Neumann entropies of reduced density matrices of the two subentities we can introduce a quantitative measure of this activity. The entropies of the two particles are shown in Fig. 7. The organism lives several units of time. Similar are the scales of time when the off-diagonal matrix elements of $\rho_{\text {int }}(t)$ become non-negligible.

Although it is clear that the "organism" behaves during the evolution as an indivisible entity, one should not confuse this indivisiblility with the so-called nonseparability discussed in quantum information theory. The organism we consider in the example is a two-qubit system and therefore one can check the separability of $\rho_{1}(t)$ by means of the Peres-Horodecki partial transposition criterion [41, 42]: A two-qubit density matrix $\rho$ is separable if and only if its partial transposition is positive. It turns out that partial transposition leaves $\rho_{1}(t)$ unchanged and thus is positive for any $t . \rho_{1}(t)$ is in this sense separable (has "zero entanglement"). This formal separability does not contradict the fact that the two-qubit organism is clearly an undivisible entity.

\section{EXAMPLES OF KINETIC EQUATIONS ASSOCIATED WITH THEIR LAX-VON NEUMANN FORM}

Let us illustrate the properties of von Neumann kinetic equations on our explicit solution (28). In our case $\tilde{f}(w)=$ $(1-s) w+s w^{2}$ with $s=s(t)=\varepsilon \cos \omega t$ [cf. Eq. (15)]. Since our solution (28) satisfies the constraint $x_{12}=x_{23}, y_{12}=y_{23}$, $p_{1}=p_{3}$, we can reduce the number of equations from six to four for the probabilities $p_{A}=x_{12}-\alpha, p_{B}=x_{13}-\beta$, 


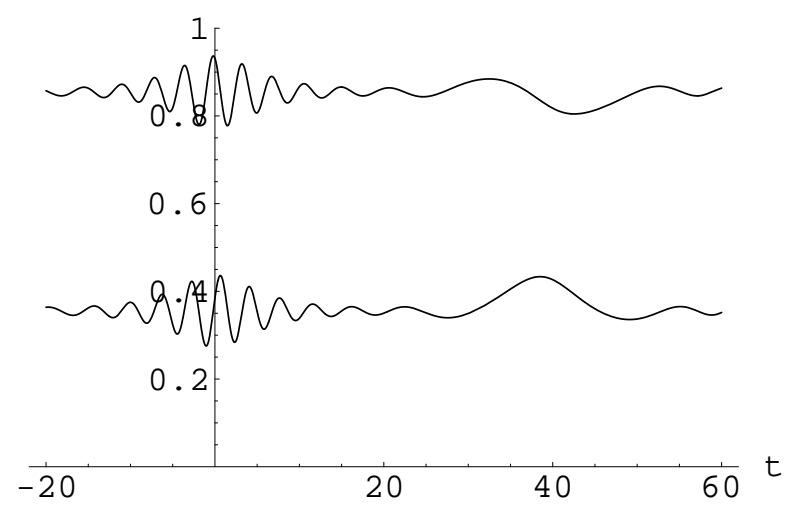

FIGURE 8. Plot of $p_{A}+1 / 2$ (upper, shifted by $1 / 2$ for clarity of the plot) and $p_{C}$ (lower), for the same parameters as in Fig. 2 .

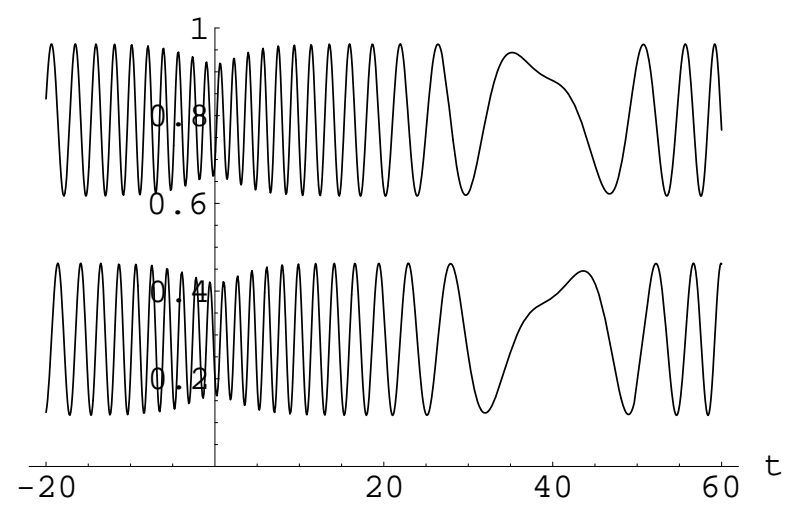

FIGURE 9. Plot of $p_{B}+1 / 2$ (upper) and $p_{D}$ (lower), for the same parameters as in Fig. 2 .

$p_{C}=y_{12}-\alpha, p_{D}=y_{13}-\beta, \alpha=-\left(1-p_{1}\right) / 2, \beta=-p_{1}$, satisfying

$$
\begin{aligned}
& \dot{p}_{A}=\frac{1-p_{1}}{2}-p_{C}+s\left(-\frac{1-p_{1}}{2} p_{1}+p_{1} p_{A}-\frac{1-p_{1}}{2} p_{B}+\frac{1-p_{1}}{2} p_{D}+p_{B} p_{C}-p_{D} p_{A}\right) \\
& \dot{p}_{B}=2 p_{1}-2 p_{D}+2 s\left(-p_{1}-\frac{\left(p_{1}+1\right)\left(1-3 p_{1}\right)}{2}+\left(1-p_{1}\right) p_{A}+\left(1-p_{1}\right) p_{C}+p_{2} p_{D}-2 p_{A} p_{C}\right) \\
& \dot{p}_{C}=-\frac{1-p_{1}}{2}+p_{A}+s\left(3 \frac{1-p_{1}}{2} p_{1}-2 p_{1} p_{A}-\frac{1-p_{1}}{2} p_{B}-p_{1} p_{C}-\frac{1-p_{1}}{2} p_{D}+p_{A} p_{B}+p_{C} p_{D}\right) \\
& \dot{p}_{D}=-2 p_{1}+2 p_{B}+2 s\left(p_{2} p_{1}-\left(1-p_{1}\right) p_{A}-p_{2} p_{B}+\left(1-p_{1}\right) p_{C}+p_{A}^{2}-p_{C}^{2}\right)
\end{aligned}
$$

As we have explained, the time dependence of $s(t)$ is a result of interaction between two parts of the system. The whole system is described by a greater number of variables, but the type of nonlinearity is qualitatively the same as for the subsystem.

Fig. 8 and Fig. 9 show that $p_{A}, p_{B}, p_{C}, p_{D}$, are greater than 0 and smaller than 1 , as expected on general grounds. These four probabilities correspond to noncommuting propositions and this is why their sum is not equal to 1 (actually, $p_{A}+p_{B}+p_{C}+p_{D}$ is time-dependent).

\section{CONCLUSIONS}

Formally nonclassical probabilistic and logical systems occur in domains that have nothing to do with quantum mechanics. Also the forms of dynamics usually known under the names of von Neumann or Heisenberg equations are typical of all the systems that posess the so called Lax representations. Therefore, one should not be surprized that 
the mathematical structures one knows from quantum mechanics can be encountered in problems that are "classical" or "macroscopic". In particular, the basic equations of chemistry or quantitative biology are typically nonlinear and kinetic and one can ask if these equations possess a "Lax form" that would make them into a kind of quantum looking dynamics? The answer is: Yes, sometimes they can be mapped into nonlinear von Neumann equations. The von Neumann equations are solitonic and integrable and therefore the kinetic equations are also solitonic and integrable.

Obviously, not all the kinetic equations one writes down in biochemistry are integrable. So the link between kinetic and von Neumann equations requires further studies. Among other aspects that are worth mentioning here are the links of von Neumann-type kinetic equations to DNA. For example, even the simple linear kinetic schemes as those discussed in the Introduction naturally lead to helical structures. The self-switching whose examples have been discussed in the context of oscillations has then an interesting reinterpretation in terms of formation of open and closed states of the helices (for details cf. [16]). Moreover, it is known that that DNA consists of two strands that evolve, in certain sense, in opposite directions (leading and lagging strands). This leads to another intriguing link with von Neumann equations since their solutions possess a natural tensor product structure and may be regarded as composite systems whose parts evolve in mutually time-reflected ways. This problem will be discussed on explicit examples in a forthcoming paper.

\section{ACKNOWLEDGMENTS}

The work of MC is a part of the Polish Ministry of Scientific Research and Information Technology (solicited) project PZB-MIN-008/P03/2003. We acknowledge the support of the Flemish Fund for Scientific Research (FWO Project No. G.0335.02).

\section{REFERENCES}

1. J. von Neumann, Grundlagen Der Quantenmechanik, Springer-Verlag, Berlin, Heidelberg, New York, 1932.

2. D. Aerts, Lett. Nuovo. Cim. 34, 107 (1982).

3. D. Aerts, "The physical origin of the EPR paradox and how to violate Bell inequalities by macroscopical systems", in Symposium on the Foundations of Modern Physics: 50 years of the Einstein-Podolsky-Rosen Gedankenexperiment, edited by $\mathrm{P}$. Lathi and P. Mittelstaedt, World Scientific, Singapore, 1985, pp. 305-320.

4. I. Pitowsky, Quantum Probability - Quantum Logic, Springer Verlag, New York, 1989.

5. D. Aerts, J. Math. Phys., 27, 202 (1986).

6. M. Czachor, Found. Phys. Lett. 5, 249 (1992).

7. D. Aerts, "The origin of the non-classical character of the quantum probability model", in Information, Complexity, and Control i $n$ Quantum Physics, edited by A. Blanquiere, S. Diner and G. Lochak, Springer-Verlag, Wien-New York, 1987, pp. 77-100.

8. C. Piron, Helvetica Physica Acta, 42, 330 (1969).

9. L. Gabora and D. Aerts, Journal of Experimental and Theoretical Artificial Intelligence, 14, 327, (2002).

10. D. Aerts and L. Gabora, Kybernetes, 34, 151 (2005).

11. D. Aerts and L. Gabora, Kybernetes, 34, 176 (2005).

12. L. Gabora, E. Rosch and D. Aerts, "How context actualizes the potentiality of a concept", in Worldviews, Science and Us: Bridging Knowledge and Its Implications for Our Perspectives of the World, World Scientific, Singapore, 2005.

13. D. Aerts and M. Czachor, Journal of Physics A-Mathematical and General, 37, L123-L32, 2004.

14. S. B. Leble, and M. Czachor, Phys. Rev. E 58, 7091 (1998).

15. D. Aerts, M. Czachor, L. Gabora, M. Kuna, A. Posiewnik, J. Pykacz, and M. Syty, Phys. Rev. E 67, 051926 (2003).

16. D. Aerts, and M. Czachor, "Abstract DNA-type Systems", submitted to Nonlinearity, 2005.

17. L. Gabora and D. Aerts, Interdisciplinary Science Reviews, 30, 69 (2005).

18. S. Kauffman, Origins of Order, Oxford University Press, New York, 1993.

19. J. Boone, E. Smith, D. Dennett and T. Earle, Current Anthropology, 39, S141, 1998.

20. J. H. Schwartz, Sudden Origins, Wiley, New York, 1999.

21. F. Varela, Principles of Biological Autonomy, Elsevier/North-Holland, New York, 1979.

22. J. Chandler and G. Van de Vijver, Closure: Emergent Organizations and their Dynamics. Vol. 901 of Annals of the New York Academy of Sciences, 1999.

23. G. Kampis, Self-Modifying Systems in Biology and Cognitive Science: A New Framework for Dynamics, Information and Complexity, Pergamon Press, New York, 1991.

24. R. Rosen, Anticipatory Systems: Philosophical, Mathematical \& Methodological Foundations, Oxford, New York, 1985.

25. L. Margulis and R. Fester, Symbiosis as a Source of Evolutionary Innovation, MIT Press, Cambridge, MA, 1991.

26. N. Eldridge and S. J. Gould, "Punctuated Equilibrium: An Alternative to Phyletic Gradualism", in Models in Paleobiology, edited by T. Schopf, Freeman, Cooper and Co., San Francisco, 1973, pp. 82-115. 
27. S. A. Newman and G. B. Müller, "Morphological Evolution: Epigenetic Mechanisms", in Embryonic Encyclopedia of Life Sciences, Nature Publishing Group, London, 1999.

28. J. Cairns, J. Overbaugh and J. Miller, Nature 335, 142 (1988).

29. C. B. Krimbas, Biology and Philosophy 19(2), 185 (2004).

30. M. Czachor, Phys. Lett. A 225, 1 (1997).

31. M. Czachor, and J. Naudts, Phys. Rev. E 59, R2497 (1999).

32. N.V. Ustinov, M. Czachor, M. Kuna, and S.B. Leble, Phys. Lett. A 279, 333 (2001).

33. N. V. Ustinov and M. Czachor, "Darboux-Integrable Equations with Non-Abelian Nonlinearities", in Probing the Structure of Quantum Mechanics: Nonlinearity, Nonlocality, Computation, and Axiomatics, edited by D. Aerts, M. Czachor, and T. Durt, World Scientific, Singapore, 2002, pp. 335-353.

34. J. L. Cieśliński, M. Czachor, and N. V. Ustinov, J. Math. Phys. 44, 1763 (2003).

35. A. Goldbeter, Biochemical Oscillations and Cellular Rhythms. The Molecular Bases of Periodic and Chaotic Behaviour, Cambridge University Press, Cambridge, 1996.

36. A. Goldbeter, Nature 420, 238 (2002).

37. M. C. Moore-Ede, C. A. Fuller, F. M. Sulzman, The Clocks That Time Us: Physiology of the Circadian Timing System, Harvard University Press, Cambridge, 1982.

38. D. Gonze, J.-C. Leloup, A. Goldbeter, C.R. Acad. Sci. Paris. 323, 57 (2000).

39. D. Gonze, M. R. Roussel, A. Goldbeter, J. Theor. Biol. 214, 577 (2002).

40. D. Gonze, J. Halloy, P. Gaspard, J. Chem. Phys. 116, 10997 (2002).

41. A. Peres, Phys. Rev. Lett. 77, 1413 (1996).

42. M. Horodecki, P. Horodecki, R. Horodecki, Phys. Lett. A 223, 1 (1996). 